$$
\begin{array}{llllllll}
S & I & N & O & P & S & 1 & 5
\end{array}
$$

La plaza de toros de Toulouse ha sido construída en tres meses; tiene una capaidad para 12.500 espectadores; 49,26 $\mathrm{m}$ de diámetro interior; $90,05 \mathrm{~m}$ de diámetro xterior, y 18,50 m de altura. A excepción de los soportes principales, losas, antepechos ' nudos, el resto de la estructura se ha prefabricado en taller a pie de obra. En este rabajo se describe la obra, sus características más importantes y los métodos consructivos empleados en su ejecución.

\section{plaza de toros en Toulouse}

JEAN BARETS, ingeniero D.P. E.

$835 \cdot 7$

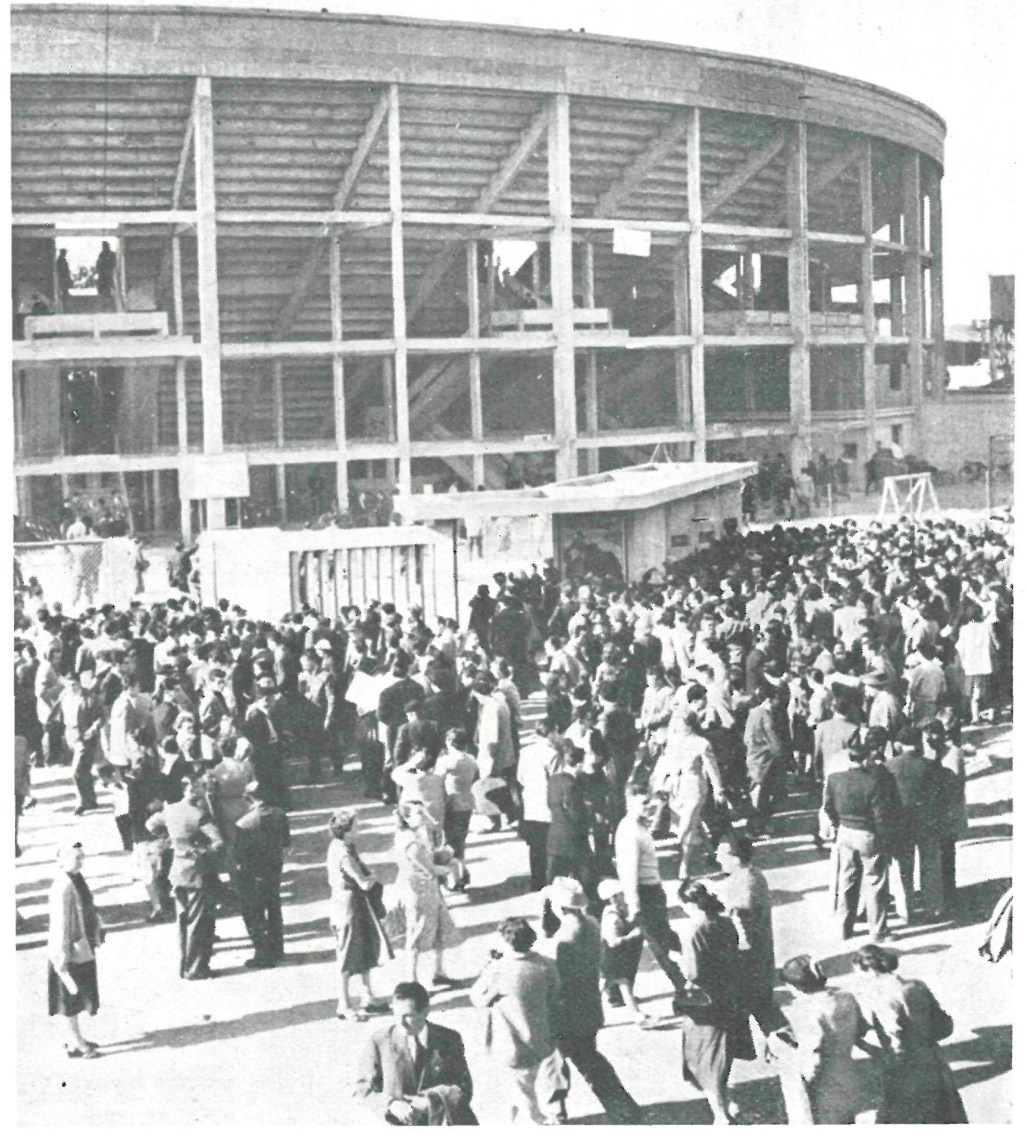

\section{eas generales}

La característica más notable de la plaza de toros de Toulouse ha to la rapidez de su ejecución. Esta obra, de tipo prefabricado, se loya sobre un terreno sano y, por tanto, no presentó problema alno de cimientos.
La plaza de toros de Toulouse, construída con elementos prefabricados
que se inauguró en el año 1953.

\section{$p l a n+a$}

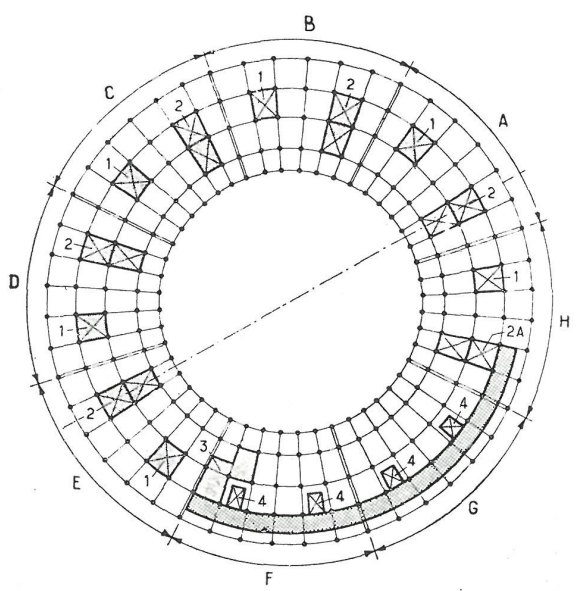

Planta de la plaza de toros. Las escaleras correspondientes a los vomitorios so único a la fila 18; los 2 , a las filas los 2 A y 3 dan acceso a la pasarela de EI segmento de anillo circular punteado re presenta la pasarela-pasillo, situada en la cota $6,42 \mathrm{~m}$.
V S T I T U T T O T E C N I C O
$D E \quad L A$
C O NSTRU
C C I ON
Y D E L
C E M ENTO 
La corona anular que forma los graderíos se ha dividido en ocho partes idénticas, separadas entre sí por medio de juntas. Cada una de estas partes se ha subdividido, a su vez, en seis partes, por lo que la estructura tiene la forma de un polígono regular de 48 lados.

\section{Superestructura}

El apoyo de los graderíos está constituído por una serie de pilares, dispuestos ordenadamente según cinco polígonos concéntricos y alineados radialmente. La sección de estos pilares es de $20 \times 25$ centímetros, a excepción de las correspondientes al polígono exterior, en el que alternan los pilares de sección normal con otros de $40 \times 60 \mathrm{~cm}$ de sección.

El conjunto de los pilares y los elementos de arriostramiento que forman las cadenas poligonales y atados radiales, constituyen el entramado o estructura resistente del coso.

Los pilares se han hormigonado "in situ", colocando los elementos del arriostramiento con sus armaduras de trabazón apoyándose sobre los encofrados, con objeto de solidarizarlos con los pilares a medida que avanza el hormigonado de éstos. Sobre los pilares se apoyan las jambas, cuya parte superior lleva los asientos de las huellas y contrahuellas de los asientos del graderío.

Las escaleras de acceso a los distintos vomitorios, también prefabricados, se apoyan directamente sobre los elementos del entramado del arriostramiento, algunos de los cuales van provistos de armaduras, que se les ha dejado en espera para unirse a las losas de los descansillos con objeto de solidarizarlos con la estructura. El tercer tramo de las escaleras se apoya, superiormente, en una viga especial, prefabricada, que se apoya sobre las jambas que soportan los bancos del graderío. Esta viga sirve de apoyo inferior de la barandilla que bordea a los vomitorios. En la zona de la planta baja, donde se han instalado los toriles y servicios, como no es posible el arranque de escaleras de acceso a los vomitorios, se ha construído una pasarela sobre esta parte, a la que se accede partiendo de las dos escaleras situadas en sus extremos. De la pasarela parten otras escaleras secundarias, que dan acceso a los graderíos superiores de esta parte del coso.

Los graderíos se han coronado con un antepecho que se ha reforzado con pequeños montantes hormigonados "in situ". Una parte superior de la plaza se ha cubierto con una marquesina de protección de espectadores. Esta marquesina, formada por una losa de hormigón, se apoya en dos filas circulares de pilares, volando sobre los pilares interiores. La cubierta se hormigonó "in situ", dando a la losa un espesor muy pequeño que le da un aspecto ligero. Los pilares interiores, de menor sección que los exteriores, no son elementos resistentes contra la acción del viento, acción que se ha reservado a la

\section{detalles constructivos}

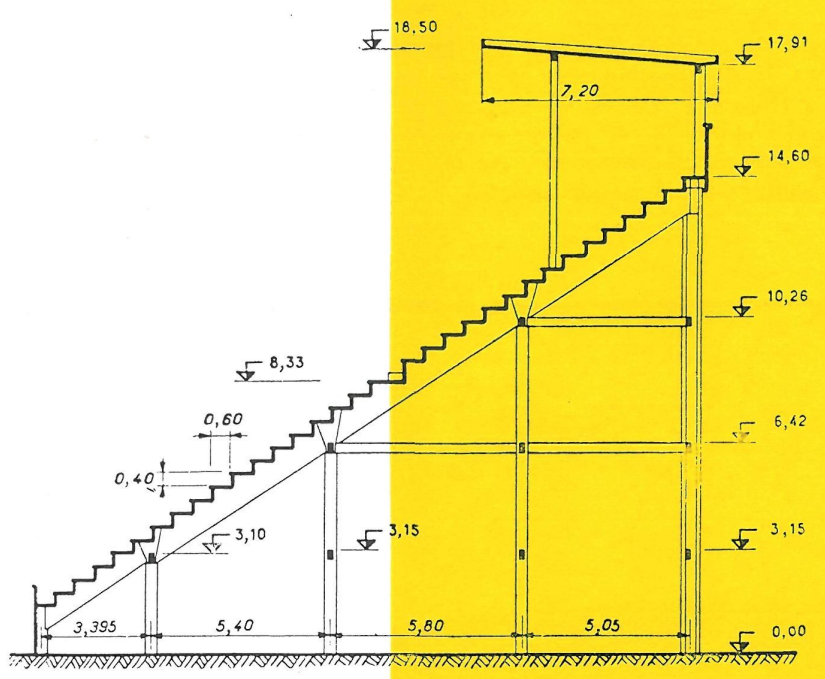

Alzado de los graderíos y marquesina.
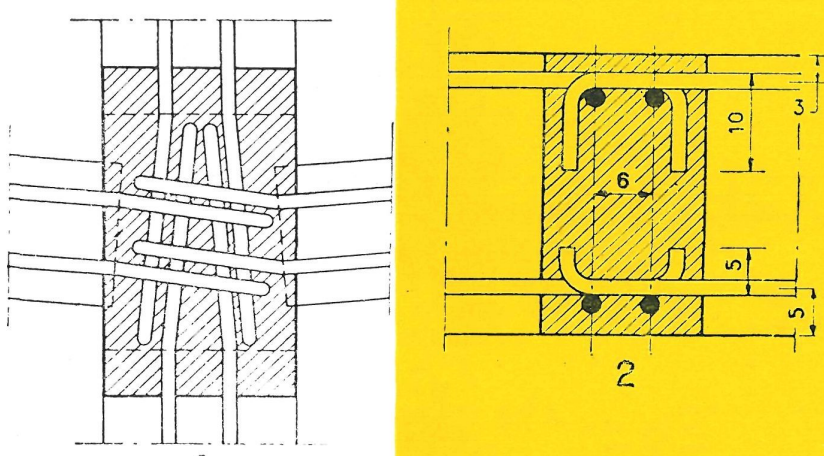

Detalle de un nudo estructural. 1. Planta.-2. Alzado.

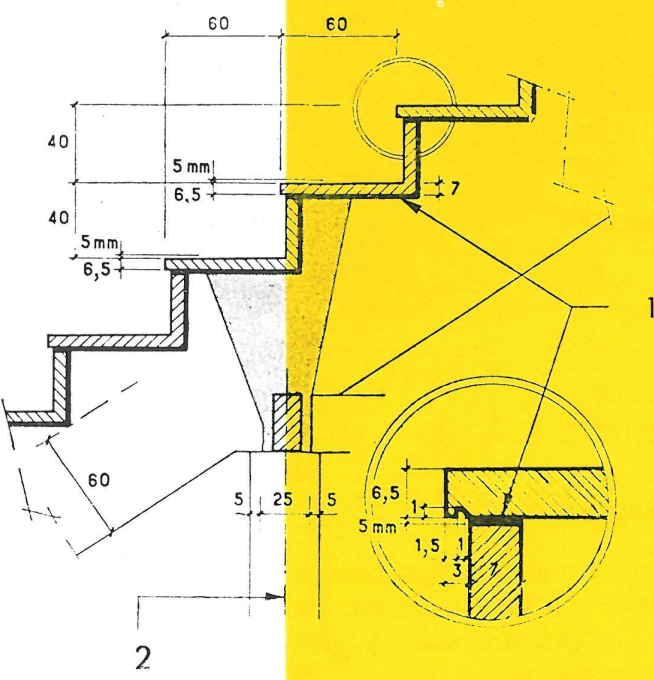

Detalle de apoyo y unión entre jambas, así como de los bancos del graderío. La parte oscura se ha hormigonado «in situ». 


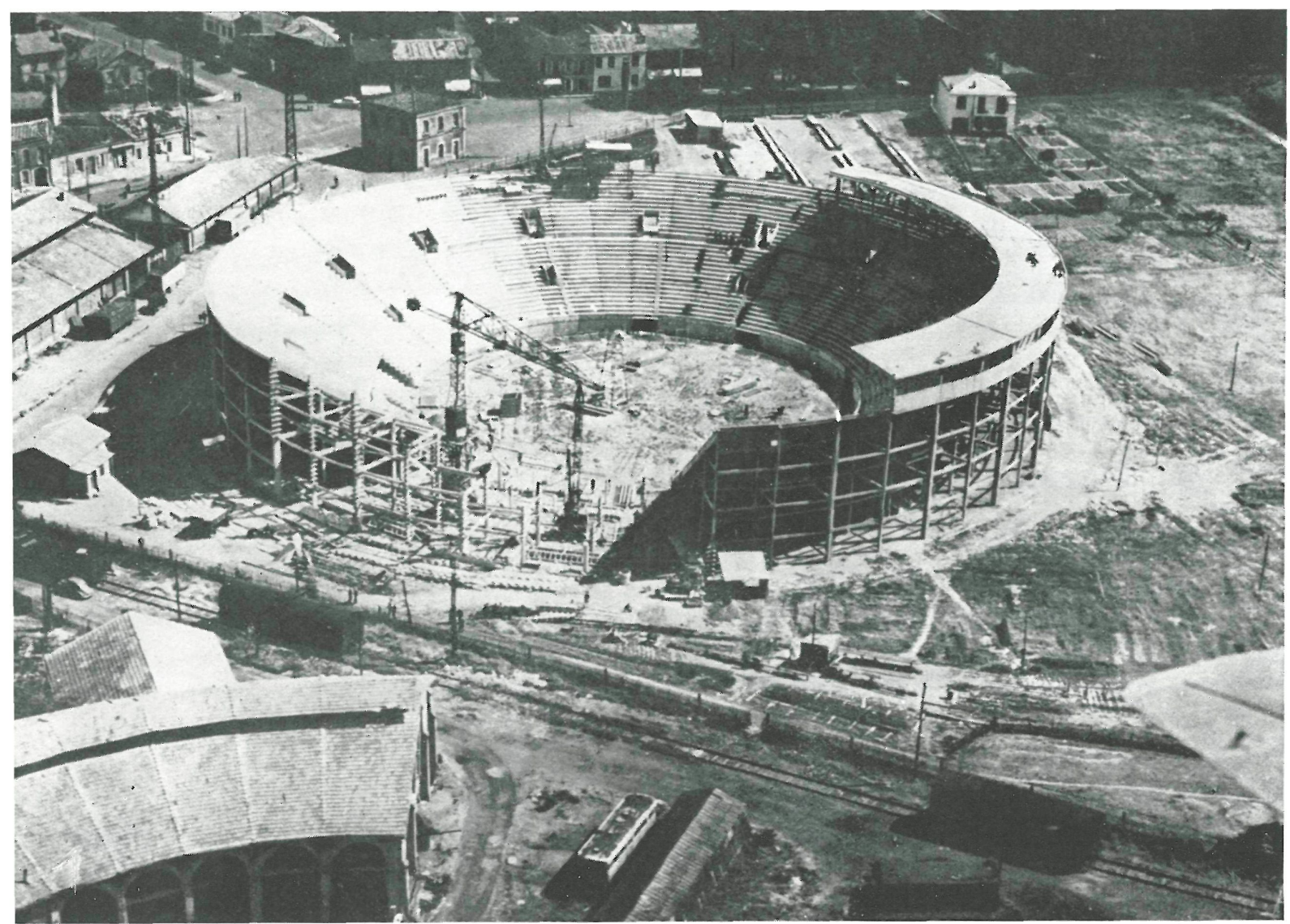

La plaza, en un estado avanzado de su construc ción.

fila exterior de pilares. La parte de maquinaria comprendida entre dos juntas consecutivas pivota sobre un punto ideal de la fila de pilares exteriores.

\section{Organización de la obra}

En las proximidades del lugar de emplazamiento de la obra se instalaron los talleres de prefabricación de elementos. Un transportador de horquilla se encargaba de recoger y transportar las piezas prefabricadas a la zona de acción de la grúa, que tenía una capacidad suficiente para elevar piezas de 2 a 3 toneladas.

La pieza más importante que se debía prefabricar era el elemento de banco para formar los graderíos. La longitud de estos elementos varía de 3 a $6 \mathrm{~m}$. La producción diaria, previamente prevista, fué de unos $120 \mathrm{~m}$ de bancos.

Para el montaje de las piezas prefabricadas, la grúa que se encargaba de su elevación y traslado se situó entre el tercer y cuarto anillo poligonal, pues un detallado estudio previo dió por resultado

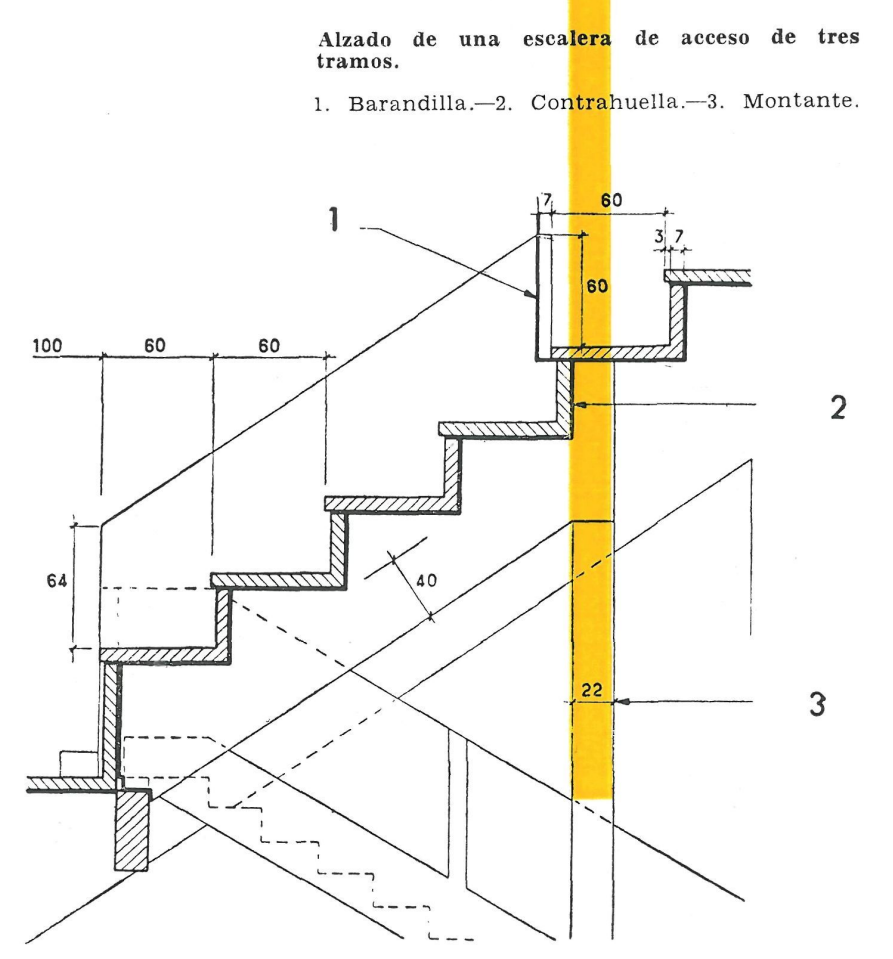

Alzado de una escalera de acceso de tres
tramos.

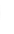




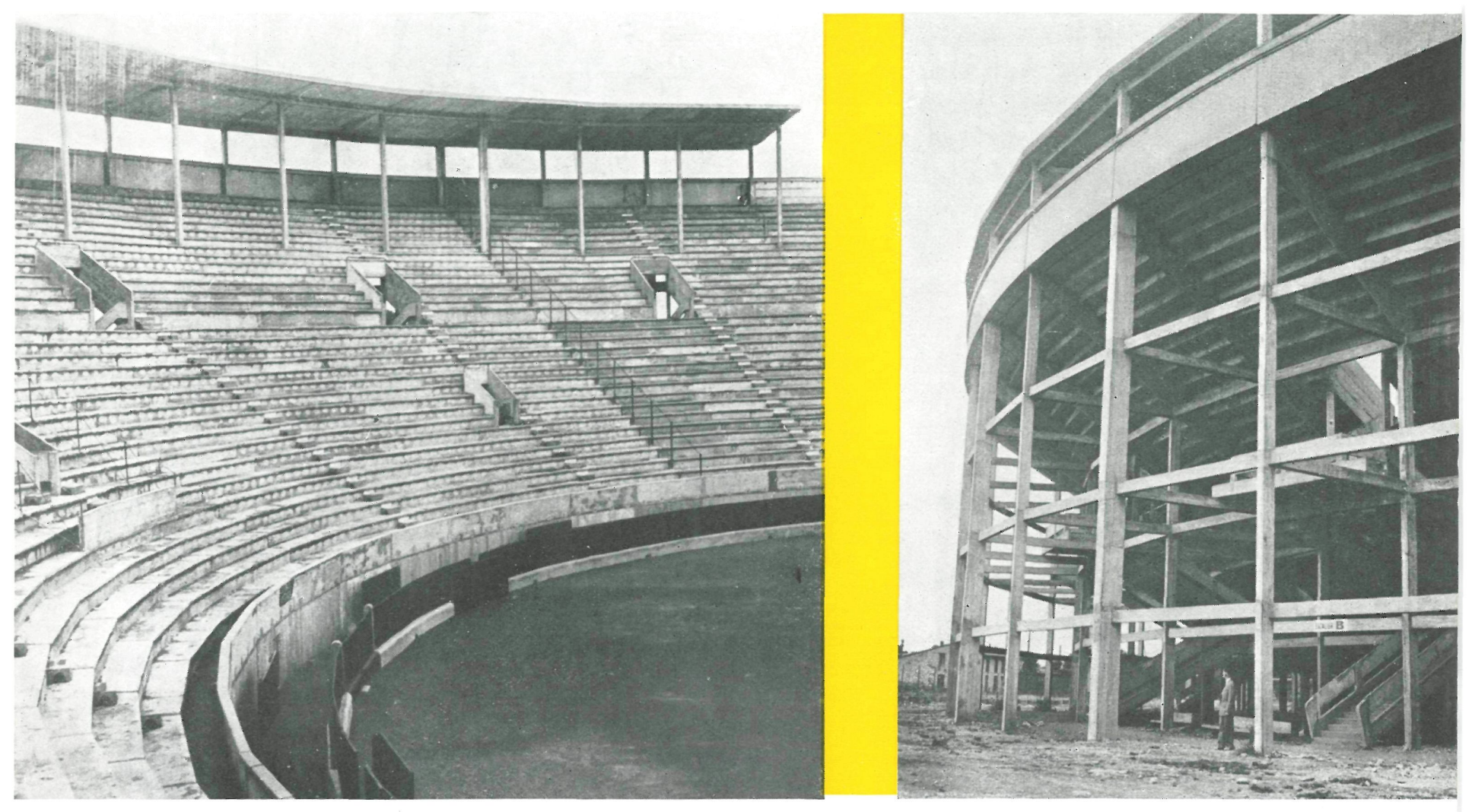

esta posición clave, ya que los elementos de arriostramiento de la estructura cerraban el paso a la libre circulación de la grúa.

\section{Ejecución}

Debido al corto plazo de tiempo disponible para la construcción, así como a la complicación que presentaba dar a los nudos el grado necesario de resistencia para seguir ininterrumpidamente la ejecución, sin que, por ello, se temiesen deformaciones inadmisibles, se recurrió al uso de cemento rápido en los casos que la cadencia de trabajo así lo exigía.
Para tener una idea del número total de piezas prefabricadas que ha sido necesario preparar para la construcción de esta plaza de toros, ha de notarse que se han fabricado 1.622 elementos para bancos de graderío, 501 vigas de arriostramiento y 270 jambas para apoyo de las piezas que forman los bancos de los graderíos.

En los servicios y anexos, corrales, enfermería y taquillas, se ha empleado fábrica de ladrillo.

J. J.U. 


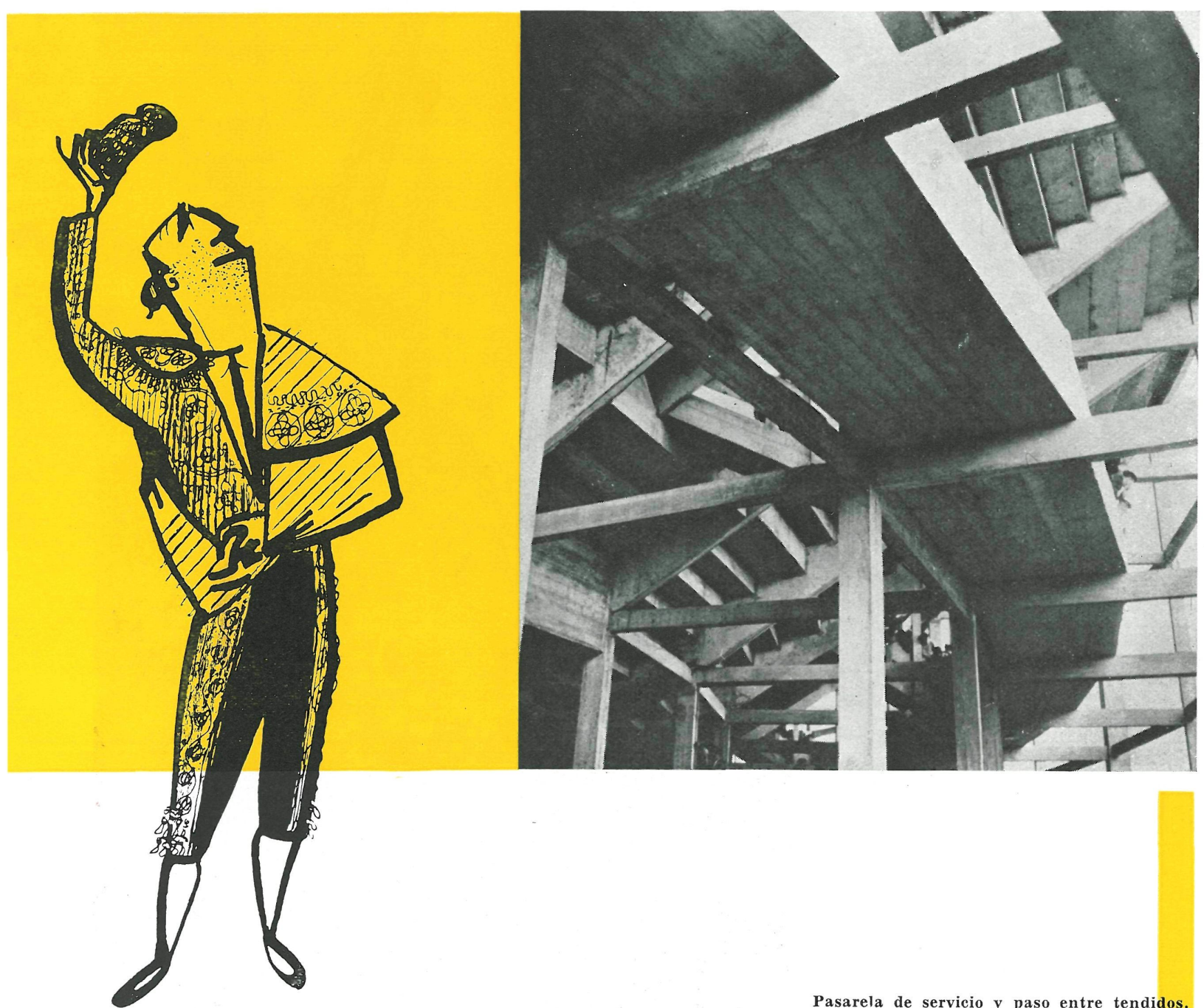

Pasarela de servicio y paso entre tendidos.

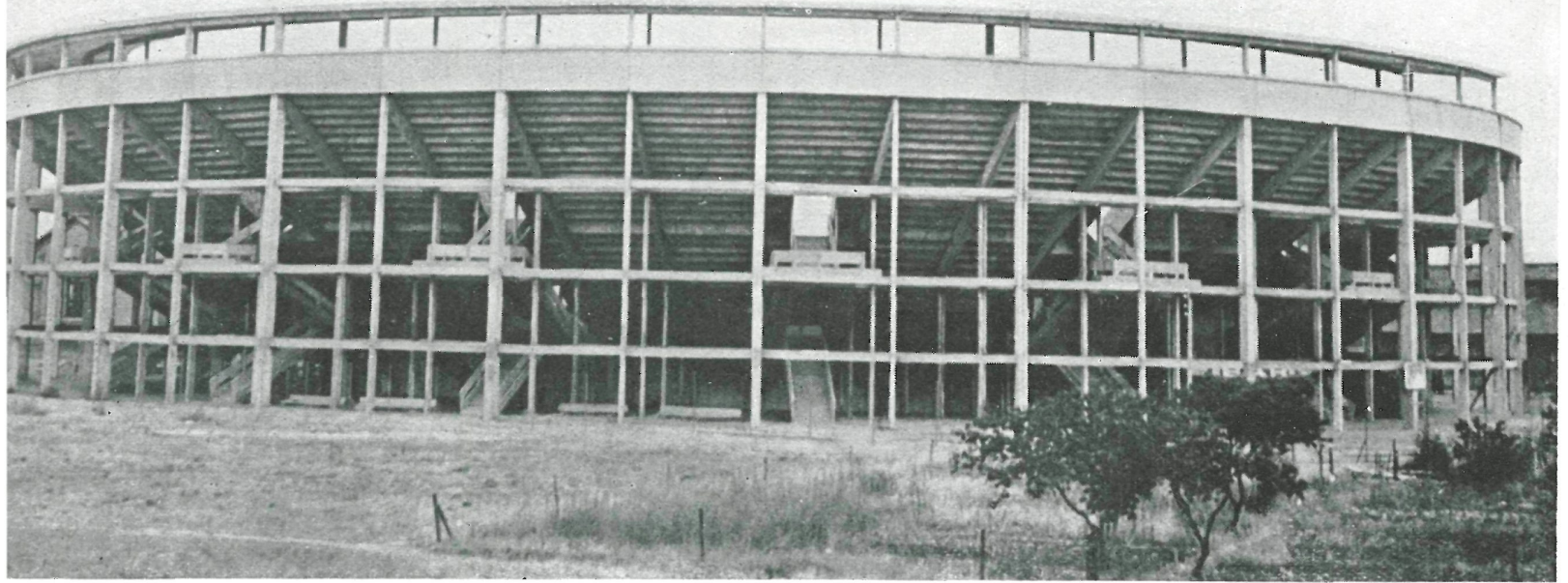

